(2) OPEN ACCESS

\title{
A cohort study comparing rate of repeat testing for sexually transmitted and blood-borne infections between clients of an internet-based testing programme and of sexually transmitted infection clinics in Vancouver, Canada
}

\author{
Mark Gilbert (D) , ${ }^{1,2}$ Travis Salway (D) ,1,2 Devon Haag, ${ }^{1}$ Elizabeth Elliot, ${ }^{3}$ \\ Christopher Fairley, ${ }^{4}$ Mel Krajden, ${ }^{1,5}$ Troy Grennan, ${ }^{1,5}$ Jean Shoveller, ${ }^{2}$ \\ Gina Suzanne Ogilvie (1) 1,2
}

${ }^{1} \mathrm{BC}$ Centre for Disease Control, Vancouver, British Columbia, Canada

${ }^{2}$ School of Population and Public Health, Faculty of Medicine, University of British Columbia, Vancouver, British Columbia, Canada

${ }^{3}$ British Columbia Colleage of Nursing Professionals, Vancouver, British Columbia, Canada

${ }^{4}$ Melbourne Sexual Health Centre, Carlton, Victoria, Australia

Department of Medicine, Faculty of Medicine, University of British Columbia, Vancouver, British Columbia, Canada

Correspondence to Dr Mark Gilbert, BC Centre for Disease Control, Vancouver, BC V5Z 4R4, Canada; mark. gilbert@bccdc.ca

Received 1 April 2019 Revised 23 July 2019 Accepted 12 August 2019 Published Online First 29 August 2019

\section{Check for updates}

(c) Author(s) (or their employer(s)) 2019. Re-use permitted under CC BY-NC. No commercial re-use. See rights and permissions. Published by BMJ.

To cite: Gilbert M,

Salway T, Haag D,

et al. Sex Transm Infect

2019:95:540-546.

\section{ABSTRACT}

Objectives Internet-based sexually transmitted and blood-borne infection (STBBI) testing services reduce testing barriers through bypassing face-to-face clinical encounters, potentially enabling clients at ongoing sexual risk to test more frequently. To our knowledge, this hypothesis has not been previously tested. We compared the frequency of repeat testing between Vancouverbased clients of GetCheckedOnline (GCO)_an internet-based STBBI testing service in British Columbia, Canada_-and clients of three sexually transmitted infection (STI) clinics in Vancouver for 29 months after GCO launched.

Methods An administrative data cohort ( $n=19497)$ was assembled using GCO, clinical and laboratory databases. We included all individuals who tested for HIV, gonorrhoea/chlamydia, syphilis or hepatitis C at three STI clinics or using GCO, between September 2014 and February 2017. The rate of repeat testing (>30 days after first episode) was compared between clients who used GCO at least once and those who tested only in STI clinics. Poisson regression was used to generate relative rate (RR) for repeat testing, with adjustment for age, gender/sexual orientation, risk factors (eg, history of STI diagnosis) and rate of testing before GCO launched.

Results 1093 GCO clients were identified, of whom 434 (40\%) had repeat test episodes; 8200/18 404 $(45 \%)$ of clinic clients tested more than once. During the 29-month analysis period, GCO clients repeat tested 1.87 times per person-year, whereas clinic clients repeat tested 1.53 times per person-year, resulting in a crude RR of 1.22 (95\% Cl: 1.14 to 1.31). Adjustment for covariates increased the RR to $1.26(95 \% \mathrm{Cl}: 1.15$ to 1.37).

Conclusions In this cohort, individuals using internetbased STBBI testing had a rate of repeat testing $22 \%$ greater than clinic-based clients. This effect was increased after adjusting for characteristics associated with higher test frequency. The online interface of GCO may facilitate more frequent testing and may therefore contribute to earlier STBBI diagnosis.

\section{BACKGROUND}

Increasing access to testing for sexually transmitted and blood-borne infections (STBBI) is a fundamental for preventing these infections. Benefits of testing are both to the individual- including timely diagnosis and treatment to prevent complications-as well as to populations by preventing secondary transmission through behaviour change, treatment to cure or reduce the risk of transmission, and partner notification. ${ }^{12}$ Increasing the uptake and frequency of testing are important, the latter for individuals at ongoing risk particularly within populations with higher STBBI prevalence such as gay, bisexual and other men who have sex with men (gbMSM), or youth. Mathematical models of gbMSM have suggested that increasing syphilis or HIV testing frequency among individuals already engaged in testing or at higher risk has greater impact on averting future infections compared with increasing testing coverage, ${ }^{3-7}$ although this is less pronounced when baseline testing levels are high. ${ }^{58}$ For example, STBBI guidelines recommend at least annual testing among gbMSM, and more frequent testing (eg, every 3 months) in individuals at higher risk. ${ }^{9} 10$

While many individuals are engaged in testing, there remains substantial variation in testing patterns according to age and risk behaviour with many falling below these test recommendations. ${ }^{11-14}$ Barriers to testing include factors related to the individual, their interaction with healthcare providers, the testing clinic, and social and structural factors. ${ }^{15}$ Among gbMSM, improving the convenience of testing may facilitate more frequent testing (eg, streamlining the testing process by reducing clinic visits through self-testing or express testing pathways). ${ }^{35}$ Indeed, two recent systematic reviews have suggested that use of HIV self-testing by gbMSM is associated with increases in HIV testing frequency. ${ }^{1617}$

Internet-based STBBI testing services are another innovation aimed at reducing testing barriers by eliminating the need for face-to-face clinical 
encounters to access testing. Individuals typically access internet-based testing by visiting a website to request a mailed self-sampling kit or receiving a laboratory form to take to a laboratory for testing, with results received online or by phone. In our prior research related to GetCheckedOnline (GCO), British Columbia's internet-based STBBI testing service, ${ }^{18}$ we demonstrated the acceptability of GCO which is valued by clients for its privacy and convenience, and use by individuals at higher risk and facing existing barriers to accessing clinical testing services. ${ }^{15} 19-21$ The development of GCO was predicated on the hypothesis that by reducing barriers, internet-based STBBI testing services have the potential to improve testing frequency among individuals at ongoing risk. ${ }^{18}$ Furthermore, we hypothesised that GCO was a complement to existing clinical testing services and individuals may test both online and in clinic-based settings (eg, depending on need for other sexual health services). Accordingly, the main objective of our study was to determine whether use of GCO was associated with higher rates of repeat testing among individuals engaged in ongoing testing through GCO and at sexually transmitted infection (STI) clinics in Vancouver, Canada. As a secondary objective, we aimed to describe patterns of overlap between use of GCO and other testing services.

\section{METHODS}

We used the Strengthening the Reporting of Observational Studies in Epidemiology statement for reporting of cohort study findings to inform our description of this study, which used a retrospective cohort design based on available administrative data between 9 September 2014 (GCO launch date) and 8 February 2017 (29 months after launch). ${ }^{22}$

\section{Intervention and settings}

The BC Centre for Disease Control (BCCDC) developed GCO which launched in Vancouver in September 2014. We previously published detailed descriptions of the GCO programme model and evaluation of its impact in Vancouver. ${ }^{15} 1821$ Individuals visiting the GCO website create an account using their email address, complete a risk assessment, create a laboratory form to take to a local specimen collection site, provide specimens, and obtain results online (if negative) or by phone (if positive). Individuals reporting symptoms or a partner with an STI are recommended to go to a clinic but may proceed. Review of results, treatment and partner notification are arranged by staff at the BCCDC provincial STI clinic. Tests offered through the service include serology for HIV, hepatitis C and syphilis; chlamydia and gonorrhoea tests are conducted on urine and self-collected throat and rectal swabs. GCO was developed as an extension of the provincial STI clinic at the BCCDC, mirroring its low-barrier clinic practice including no fees, similar test recommendations and consent processes, and no verification of client identity. Tests are performed using a system-generated unique alphanumeric code instead of first and last name (same code used each time a user tests through GCO). ${ }^{18}{ }^{23} \mathrm{GCO}$ also includes automated email testing reminders, with the default at 12 months, and at 3 months for individuals identified at higher risk of based on their risk assessment (sex partner with an STI, condomless sex with more than one or an HIV-positive partner, past STI diagnosis or using drugs with a shared needle). Individuals can opt out of or change the frequency of testing reminders, which also include a 6-month option.

To estimate the impact of GCO on increasing test frequency, we identified a population of individuals seeking STI testing that were potentially exposed to the GCO intervention. We used clients of three public health STI clinics located in close proximity to the GCO specimen collection sites in Vancouver (figure 1), including the provincial STI clinic and two community clinics located within gender and sexual minority organisations. All clinics were operated by BCCDC staff, are low-threshold and follow similar clinical procedures. GCO was promoted at all three clinic sites by staff, posters and brochures with individuals provided access codes to use the service, as well as to Vancouver gbMSM. In prior research, we found that $41 \%$ of provincial STI clinic clients are aware of GCO and 30\% of GCO clients having previously tested at the provincial STI clinic. ${ }^{15}{ }^{24}$ In the same survey, 24\% of GCO clients reported previously testing at each of the two community STI clinics (Kim Thomson, BCCDC, personal communication, 2018).

\section{Participants, data sources and measurement}

We drew on clinical, GCO and laboratory data to establish comprehensive testing histories. A common electronic medical record (EMR) was the foundational data source for this study, including data on testing and covariates for all three STI clinic clients as well as GCO client testing data. All clients with $\geq 1$ test for HIV, syphilis, chlamydia, gonorrhoea or hepatitis C during the study period and residing in the Greater Vancouver area were extracted from the EMR and deterministically linked via a common unique identifier to the GCO programme database to obtain self-reported data on covariates for GCO clients. This merged dataset comprised the total set of clients (participants) included in this cohort study. The merged data were linked via probabilistic matching on first name, last name, gender, date of birth and EMR record number to the provincial public health Laboratory Information System (LIS). The provincial public health laboratory conducts all testing for GCO and the three clinics in this study, and the majority (95\%-100\%) of HIV, hepatitis C and syphilis testing, and approximately $20 \%$ of all chlamydia and gonorrhoea testing in the province. This linkage was done to identify additional STBBI tests that may have been conducted at other clinical sites during the study period (eg, other STI clinic, family doctor's office), as well as pre-implementation STBBI tests. We examined the success of linkage of records to laboratory data to assess potential for linkage biases to influence our results.

\section{Variables}

Our exposure of interest was any use of GCO (GCO clients), defined as testing at least once using GCO during the study period (ie, regardless of whether tested through an STI clinic or not). Our outcome of interest was the rate of repeat testing during the study period. A repeat test episode was defined as any test episode occurring $>30$ days after a first test episode with GCO or a STI clinic. A test episode was defined as a GCO or clinic visit that included any test for HIV, gonorrhoea, chlamydia, syphilis or hepatitis C. Additional tests completed $<30$ days after any test were assumed to be part of the same test episode (eg, repeat tests related to indeterminate results or problems with sample).

We considered the following covariates to potentially affect both the decision to use GCO and an individual's rate of testing: age, gender/sexual orientation, reporting symptoms at least once, testing due to a partner being diagnosed with an STI at least once and known rate of testing prior to the launch of GCO (ie, pre-implementation, defined as the number of test episodes during the 29-month period preceding GCO launch, 9 April 2012-8 September 2014, divided by person-years, that is, time 


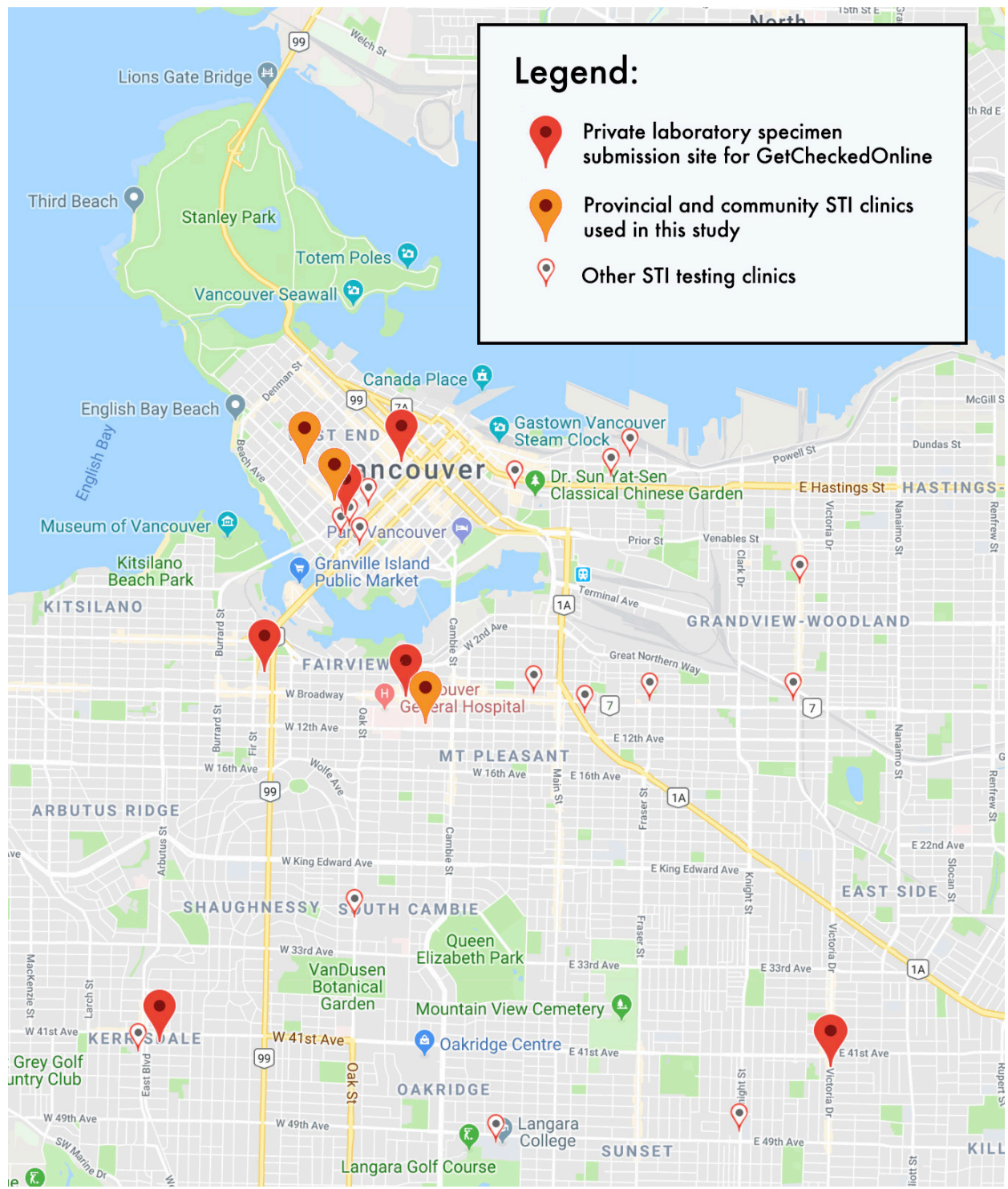

Figure 1 Map showing locations of study clinics, specimen collection sites and other sexually transmitted infection clinics.

from first episode to 8 September 2014). We also included two covariates considered theoretically important: having at least one HIV-positive partner (past 3 months, for GCO clients, or currently, for clinic clients), and prior STI diagnosis (past year, for GCO clients, or ever, for clinic clients). Gender/sexual orientation was defined based on client gender and gender of sexual partners identified during the study period: women (female gender), men who have sex with women (MSW; male gender and female sex partners only) and men who have sex with men (MSM; male gender and at least one male sex partner reported during the study period). Ethnicity was excluded due to high missingness (12\% among GCO clients, 26\% for STI clinic clients).

\section{Statistical methods}

Our primary analyses included four steps. First, we described the overlap in client use of GCO and STI clinics. Second, we compared the characteristics of GCO and clinic client groups using t-test and $\chi^{2}$ tests as appropriate (level of significance $\mathrm{p}<0.05)$. Third, we estimated the rate of repeat testing by use of GCO and covariates. We calculated the rate of repeat testing as the number of repeat test episodes divided by person-year of observation, calculated as the date of the first recorded GCO visit for GCO clients or STI clinic episode for clinic clients following launch of GCO (8 September 2014) to the end of the study period (8 February 2017). For example, if an individual first tested through GCO on 1 January 2015, then in a study STI clinic on 1 January 2016 leading to a test on 15 January 2016 to resolve an indeterminate result, and yet again through GCO on 1 January 2017, this individual was classified as a GCO client with a repeat testing rate of 0.95 (2 repeat test episodes/2.11 person-years). As our exposure of interest was repeat testing rate among individuals engaged in ongoing testing, we excluded individuals having a single test only.

Fourth, we used Poisson regression to model the association between groups and rate of repeat testing, with adjustment for all covariates. Pre-implementation test rate was log-transformed to ensure normality. All analyses were completed using R V.3.4.1.

\section{RESULTS}

In total, 19497 clients were included in the analysis: 1093 (5.6\%) GCO clients comprising 1951 test episodes and 18404 (94.4\%) clinic clients comprising 39357 test episodes. Most GCO clients (821/1093, 75\%) tested only through GCO; 272/1093 (25\%) 


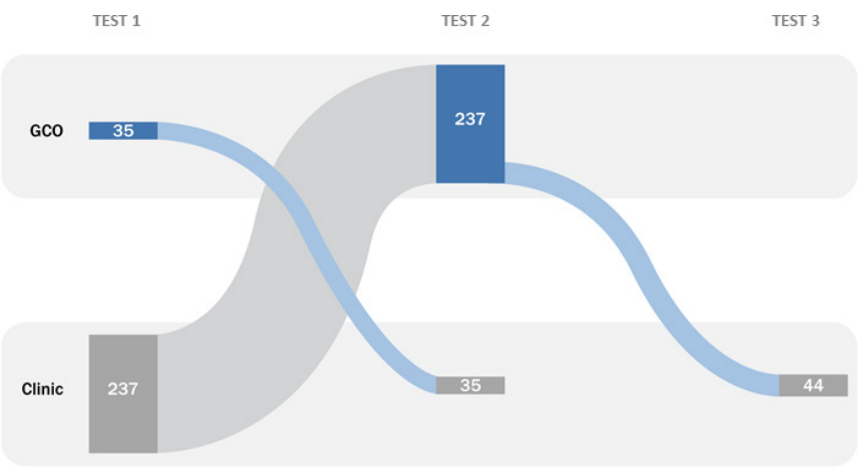

Figure 2 Sankey diagram demonstrating the order of testing for 272 clients in the GCO group identified as testing in both sexually transmitted infection clinic sites and through GCO during the study period. GCO, GetCheckedOnline

also tested at one of the three STI clinics. Of these 272 clients, $237(87 \%)$ first tested at an STI clinic, and then tested using GCO (of which a further 44 subsequently repeat tested in an STI clinic). The remaining 35 (13\%) first tested using GCO and then tested at an STI clinic (figure 2).

Compared to STI clinic clients, GCO clients included more individuals 30-39 years of age, white individuals, women, MSW, clients with a prior STI and clients reporting HIV-positive partners, and fewer MSM (all $\mathrm{p}<0.05$ ) (table 1$)$. The proportion of clients reporting symptoms $(\mathrm{p}=0.06)$ or contact with a partner with an STI $(p=0.53)$ and the pre-implementation testing rate $(p=0.85)$ were not significantly different. A comparable proportion of clients were linked to LIS records during the pre-implementation period $(\mathrm{p}=0.82)$. A greater proportion of GCO clients were linked to LIS records representing tests at other clinics during the study period $(\mathrm{p}<0.0001)$.

Sixty per cent $(659 / 1093)$ of GCO clients and $55 \%$ $(10,204 / 18,404)$ of clinic clients tested once during the study period and were excluded from the analysis. The remaining 434 GCO clients repeat tested 1.87 times per person-year with an average 1.07 person-years of follow-up; the remaining 8200 clinic clients repeat tested 1.53 times per person-year with an average 1.67 person-years of follow-up. The unadjusted relative rate (RR) of repeat testing was 1.22 times higher (95\% CI: 1.14 to 1.31 ) among GCO clients than among clinic clients. The RR of repeat testing for GCO clients remained significantly higher on adjustment for covariates at 1.26 (95\% CI: 1.15 to 1.37 ) (table 2).

\section{DISCUSSION}

We found that clients engaged in ongoing testing and using GCO at least once during the study period had a rate of repeat testing $22 \%$ greater than clients testing in STI clinics only, an effect that increased slightly after adjusting for relevant covariates. We also observed that a small number of clients testing through GCO had subsequent tests at one of the STI clinics included in our study. These results support our hypotheses that internet-based STBBI testing services can lead to increases in testing frequency (which is one of GCO's programme objectives), ${ }^{18}$ and are complementary to face-to-face clinical services. The methodological strengths of

Table 1 Characteristics of GCO and STI clinic client groups

\begin{tabular}{|c|c|c|c|}
\hline & GCO clients $n=1093$ & STI clients $n=18404$ & $P$ value* \\
\hline Age (years), mean, median (IQR) & $34.4,32.2(27.0,39.7)$ & $34.0,31.0(26.0,40.0)$ & 0.2098 \\
\hline$<30$ & $440(40.3 \%)$ & $7973(43.3 \%)$ & 0.0504 \\
\hline $30-39$ & $387(35.4 \%)$ & $5808(31.6 \%)$ & 0.0087 \\
\hline $40+$ & $266(24.3 \%)$ & $4621(25.1 \%)$ & 0.5918 \\
\hline Missing & $0 / 1093(0.0 \%)$ & $2(<0.1 \%)$ & $\mathrm{n} / \mathrm{c}$ \\
\hline \multicolumn{4}{|l|}{ Gender/sexual orientation } \\
\hline Women & $307(28.1 \%)$ & $4271(23.2 \%)$ & 0.0003 \\
\hline MSM & $397(35.0 \%)$ & $6071(41.6 \%)$ & 0.0250 \\
\hline MSW & $383(36.3 \%)$ & $7659(33.0 \%)$ & $<0.0001$ \\
\hline Missing & $6(0.5 \%)$ & $403(2.2 \%)$ & 0.0004 \\
\hline \multicolumn{4}{|l|}{ Ethnicity } \\
\hline White & $688(62.9 \%)$ & $8743(47.5 \%)$ & $<0.0001$ \\
\hline Non-white/racialised & $274(25.1 \%)$ & $4893(26.6 \%)$ & 0.2848 \\
\hline Missing & $131(12.0 \%)$ & $4768(25.9 \%)$ & $<0.0001$ \\
\hline Symptoms $†$ & $245(22.4 \%)$ & $4600(25.0 \%)$ & 0.0600 \\
\hline Partner contact to STI† & $144(13.2 \%)$ & $2556(13.9 \%)$ & 0.5363 \\
\hline History of STI diagnosist & $237(21.7 \%)$ & $3203(17.4 \%)$ & 0.0004 \\
\hline HIV-positive partnert & $38(3.4 \%)$ & $425(2.3 \%)$ & 0.0183 \\
\hline \multicolumn{4}{|l|}{ Clients linked to LIS record (excluding STI clinic test episodes) } \\
\hline During pre-implementation period & $464(42.4 \%)$ & $7900(42.9 \%)$ & 0.8181 \\
\hline During study period (ie, tests at other clinics) & $560(51.2 \%)$ & $6717(36.5 \%)$ & $<0.0001$ \\
\hline Pre-implementation rate of testing per person-year mean, median (IQR) & $3.29,1.64(0.98,2.66)$ & $3.16,1.71(1.00,2.91)$ & 0.8527 \\
\hline \multicolumn{4}{|l|}{ Number of test episodes } \\
\hline 1 & $659(60.3 \%)$ & $10204(55.4 \%)$ & 0.0019 \\
\hline 2 or more (ie, repeat tester) & $434(39.7 \%)$ & $8200(44.6 \%)$ & \\
\hline
\end{tabular}

* Level of significance set at $p<0.05$ based on $\mathrm{t}$-test or $\chi^{2}$ test.

tReported in at least one visit (during study period).

GCO, GetCheckedOnline; LIS, laboratory information system; MSM, men who have sex with men; MSW, men who have sex with women; n/c, not calculated; STI, sexually transmitted infection. 
Table 2 Variables associated with repeat testing rate, restricted to clients reporting two or more test episodes (434 GC0 clients, and 8200 clinic clients)

\begin{tabular}{|c|c|c|c|c|c|}
\hline & $\begin{array}{l}\text { Number of test } \\
\text { episodes }\end{array}$ & $\begin{array}{l}\text { Number of } \\
\text { person-years }\end{array}$ & $\begin{array}{l}\text { Repeat testing rate } \\
\text { (per person-year) }\end{array}$ & $\begin{array}{l}\text { Unadjusted } \\
\text { RR }(95 \% \mathrm{Cl})\end{array}$ & $\begin{array}{l}\text { Adjusted } \\
\text { RR }(95 \% \mathrm{Cl})\end{array}$ \\
\hline \multicolumn{6}{|l|}{ Client group } \\
\hline STI clinics & 20953 & 13666 & 1.53 & Reference & Reference \\
\hline \multicolumn{6}{|l|}{ Age (years) } \\
\hline$<30$ years & 8520 & 5845 & 1.46 & Reference & Reference \\
\hline \multicolumn{6}{|l|}{ Gender/sexual orientation } \\
\hline Women & 3104 & 2872 & 1.08 & Reference & Reference \\
\hline MSM & 15166 & 7869 & 1.93 & 1.78 (1.72 to 1.85$)$ & 1.96 (1.83 to 2.03$)$ \\
\hline MSW & 3243 & 3218 & 1.01 & 0.93 (0.89 to 0.98 ) & 0.82 (0.77 to 0.87$)$ \\
\hline \multicolumn{6}{|l|}{ Symptoms } \\
\hline Yes & 6232 & 2876 & 2.17 & 1.56 (1.52 to 1.61$)$ & 1.43 (1.38 to 1.49$)$ \\
\hline \multicolumn{6}{|l|}{ History of STI diagnosis } \\
\hline No & 15665 & 10583 & 1.48 & Reference & Reference \\
\hline Yes & 6146 & 3542 & 1.74 & 1.17 (1.14 to 1.21$)$ & 1.13 (1.09 to 1.17$)$ \\
\hline \multicolumn{6}{|l|}{ HIV-positive partner } \\
\hline No & 20013 & 13486 & 1.48 & Reference & Reference \\
\hline Yes & 1798 & 638 & 2.82 & 1.90 (1.81 to 1.99$)$ & 1.48 (1.40 to 1.56$)$ \\
\hline $\begin{array}{l}\text { Pre-implementation rate of } \\
\text { testing (log-transformed) }\end{array}$ & - & - & - & 1.36 (1.34 to 1.39$)$ & 1.26 (1.23 to 1.28$)$ \\
\hline
\end{tabular}

GCO, GetCheckedOnline; MSM, men who have sex with men; MSW, men who have sex with women; RR, relative rate; STI, sexually transmitted infection.

our study included longitudinal testing data for 19497 individuals followed over 29 months following GCO implementation; we also linked study data to provincial laboratory data to describe pre-implementation testing rate and capture tests conducted at other clinics.

This observational study relied on routinely collected GCO programme and clinic EMR data, and selected covariates based on data considered most comparable between these datasets. As such, unmeasured confounders are possible as are explanatory variables potentially related to STBBI testing frequency that we were unable to directly assess, such as direct measures of sexual behaviour that determines risk of infection, relying instead on proxy measures, such as partner with an HIV and history of STI diagnosis. The exclusion of ethnicity due to missing data may also have biassed our results, as it is plausibly associated with repeat testing rate. We also included data that were collected in different ways, being self-reported in GCO programme data and provider documented in the EMR and with the latter likely affected by social desirability bias. Moreover, history of STI diagnosis was measured using different recall periods between GCO and clinic, which may have influenced our results. To address this limitation, we re-ran the multivariable model excluding the history of STI diagnosis covariate, yielding a comparable RR of 1.28 (95\% CI: 1.17 to 1.39 ) comparing GCO to clinic. Clinic clients had longer periods of observation than GCO clients, likely owing to the gradual uptake of GCO during the study period. As a result, the GCO group may have included disproportionately fewer repeat test episodes among clients who have a lower STBBI risk and therefore a longer inter-test interval, biassing our effect measure of interest (RR comparing GCO vs clinic) towards the null. Due to the nature of data used, we were also unable to ascertain losses to follow-up due to individuals moving out of the Vancouver area; however, these are likely minimal, given the relatively short period of follow-up, and we do not expect these losses to differ by exposure group.

There are few comparative studies of users and non-users of internet-based STBBI testing services, highlighting the need for this and other studies. ${ }^{15} 25$ We are not aware of other studies investigating the hypothesis that internet-based STBBI testing services facilitate increased frequency of testing. Two randomised controlled trials have suggested that internet-based self-sampling kits for chlamydia testing lead to increased short-term testing uptake among youth in primary care settings, and uptake of re-testing following a chlamydia diagnosis (in combination with test reminders). ${ }^{26}{ }^{27}$ While our findings suggest that when used by clients, GCO facilitates higher frequency of STBBI testing among individuals engaged in ongoing testing, the exact pathways driving this increased testing frequency remain unclear. These results may reflect the improved convenience of testing and privacy reported by GCO clients. ${ }^{15} 28$ GCO clients have also described how the regular reminder emails to test increased their testing frequency and understandings of ongoing risk ${ }^{28}$ which is reflected in trial evidence. ${ }^{29}{ }^{30} \mathrm{We}$ also observed that a small number of clients testing through GCO subsequently tested at STI clinics. It is possible that these clients may have decided to no longer test through GCO and to return to clinic-based testing. It is also possible that this may reflect preferences for GCO or STI clinic testing in different scenarios; in interviews with gbMSM users of GCO, many continued to value clinic services and anticipated using both services in the future. ${ }^{28}$ Currently, approximately $40 \%$ of all tests completed through GCO 
are in clients who have tested repeatedly. Further study of longitudinal testing patterns of GCO users would help shed further insight into the roles that online and clinic-based sexual health services play in increasing overall access to testing services. Further research would also be helpful in understanding why GCO users test while symptomatic or as a contact despite recommendations, as this may delay accessing syndromic treatment. It is possible that the same barriers to accessing clinic-based testing among GCO users may also affect willingness to present for care in this situation.

In conclusion, the finding that clients engaged in ongoing testing using GCO had a higher rate of repeat testing compared with STI clinic clients provides evidence in support of our hypothesis that internet-based STBBI testing services facilitate increases in testing frequency through reduction of testing barriers. We also found evidence to support our hypothesis that when the option is available, individuals may choose to test both online and in clinical settings. Our findings are specific to GCO and British Columbia; given the diversity of internet-based STBBI testing models, further studies testing these hypotheses are needed. It is also important to consider, through mathematical modelling and economic analysis studies, whether increases in testing frequency due to interventions such as GCO have a true population impact by leading to earlier diagnosis and prevention of secondary infections, and are cost-effective. $^{8}$

\section{Key messages}

- By improving convenience and reducing testing barriers, internet-based testing services for sexually transmitted and blood-borne infections may increase testing frequency for individuals at ongoing risk.

- No prior study has tested this hypothesis, and few studies have considered how individuals may use both internetbased and clinic-based testing services.

- We found a higher rate of repeat testing among clients of British Columbia's GetCheckedOnline programme compared with clients of sexually transmitted infection (STI) clinics in Vancouver.

- This study also suggests that when both options are available, some individuals may test both online and in STI clinics

\section{Handling editor Claudia S Estcourt}

Acknowledgements We would like to acknowledge the support provided by Paul Kim and Stanley Wong, BC Centre for Disease Control in the preparation of the data extract used for this analysis.

Contributors MG is the principal investigator of this study and led the overall conduct of the study and manuscript preparation. TS conducted analyses and contributed to manuscript preparation. DH and MK assisted with securing data, preparation and analysis. TS, EE, MG, CKF, MK, JS and GO contributed to the study design and interpretation of study findings. All authors have reviewed and contributed to the manuscript.

Funding Funding for this study was provided by the Canadian Institutes of Health Research (grants PHE-114129, PHE-318068), which played no other role in this study.

Competing interests $T S, D H, M K, J S, G O$, and MG report the above-named grants from Canadian Institutes of Health Research, during the conduct of the study.

Patient consent for publication This study used available health administrative data and individual patient consent was not required. Use of GetCheckedOnline data for this study was consistent with the terms of use agreed to by all users of the service.

Ethics approval Ethics approval for this study was obtained from the research ethics board at the University of British Columbia (approval number H11-01168).

Provenance and peer review Not commissioned; externally peer reviewed.
Open access This is an open access article distributed in accordance with the Creative Commons Attribution Non Commercial (CC BY-NC 4.0) license, which permits others to distribute, remix, adapt, build upon this work non-commercially, and license their derivative works on different terms, provided the original work is properly cited, appropriate credit is given, any changes made indicated, and the use is non-commercial. See: http://creativecommons.org/licenses/by-nc/4.0/.

\section{ORCID iDs}

Mark Gilbert http://orcid.org/0000-0001-5978-6843

Travis Salway http://orcid.org/0000-0002-5699-5444

Gina Suzanne Ogilvie http://orcid.org/0000-0001-5783-4493

\section{REFERENCES}

1 Global health sector strategy on sexually transmitted infections 2016-2021. Geneva, Switzerland: World Health Organization, 2016

2 90-90-90. An ambitious treatment target to help end the AIDS epidemic. UNAIDS 2014.

3 Down I, Wilson DP, McCann PD, et al. Increasing gay men's testing rates and enhancing partner notification can reduce the incidence of syphilis. Sex Health 2012;9:472-80.

4 Gray RT, Hoare A, Prestage GP, et al. Frequent testing of highly sexually active gay men is required to control syphilis. Sex Transm Dis 2010;37:1-305.

5 Gray RT, Prestage GP, Down I, et al. Increased HIV testing will modestly reduce HIV incidence among gay men in NSW and would be acceptable if HIV testing becomes convenient. PLoS One 2013:8:e55449.

6 Tuite AR, Fisman DN, Mishra S. Screen more or screen more often? using mathematical models to inform syphilis control strategies. BMC Public Health 2013;13:606.

7 Wilson DP, Hoare A, Regan DG, et al. Importance of promoting HIV testing for preventing secondary transmissions: modelling the Australian HIV epidemic among men who have sex with men. Sex Health 2009;6:19-33.

8 Irvine MA, Konrad BP, Michelow W, et al. A novel Bayesian approach to predicting reductions in HIV incidence following increased testing interventions among gay, bisexual and other men who have sex with men in Vancouver, Canada. J $R$ Soc Interface 2018;15.

9 Clutterbuck D, Asboe D, Barber T, et al. 2016 United Kingdom national guideline on the sexual health care of men who have sex with men. Int J STD AIDS 2018;15.

10 Canadian guidelines on sexually transmitted infections, 2019. Available: https:// www.canada.ca/en/public-health/services/infectious-diseases/sexual-health-sexuallytransmitted-infections/canadian-guidelines/sexually-transmitted-infections.html [Accessed 3 Jul 2019].

11 Katz DA, Dombrowski JC, Swanson F, et al. Hiv intertest interval among MSM in King County, Washington. Sex Transm Infect 2013:89:32-7.

12 Gilbert M, Hottes TS, Lester $\mathrm{R}$, et al. Time since last negative HIV test among men who have sex with men and people who use injection drugs in British Columbia, 2006-2011. Can J Public Health 2014;105:e63-8.

13 An Q, Song R, Finlayson TJ, et al. Estimated HIV Inter-test interval among people at high risk for HIV infection in the U.S. Am J Prev Med 2017;53:355-62.

14 McDaid LM, Aghaizu A, Frankis J, et al. Frequency of HIV testing among gay and bisexual men in the UK: implications for HIV prevention. HIV Med 2016;17:683-93.

15 Gilbert M, Thomson K, Salway T, et al. Differences in experiences of barriers to STI testing between clients of the Internet-based diagnostic testing service GetCheckedOnline.com and an STI clinic in Vancouver, Canada. Sex Transm Infect 2019:95:151-6.

16 Zhang C, Li X, Brecht M-L, et al. Can self-testing increase HIV testing among men who have sex with men: a systematic review and meta-analysis. PLoS One 2017;12:e0188890

17 Johnson CC, Kennedy C, Fonner V, et al. Examining the effects of HIV self-testing compared to standard HIV testing services: a systematic review and meta-analysis. $J$ Int AIDS Soc 2017;20:21594.

18 Gilbert M, Haag D, Hottes TS, et al. Get Checked... where? the development of a comprehensive, integrated Internet-based testing program for sexually transmitted and blood-borne infections in British Columbia, Canada. JMIR Res Protoc 2016:5:e186.

19 Hottes TS, Farrell J, Bondyra M, et al. Internet-Based HIV and sexually transmitted infection testing in British Columbia, Canada: opinions and expectations of prospective clients. J Med Internet Res 2012;14:e41.

20 Gilbert M, Thomson $\mathrm{K}$, Chabot $\mathrm{C}$, et al. Online versus in-person testing: a qualitative analysis of testing preferences among youth and men who have sex with men using an online HIV/STI testing service in Vancouver Canada 2017.

21 Gilbert M, Salway T, Haag D, et al. Use of GetCheckedOnline, a comprehensive web-based testing service for sexually transmitted and blood-borne infections. J Med Internet Res 2017:19:e81.

22 von Elm E, Altman DG, Egger $M$, et al. The strengthening the reporting of observational studies in epidemiology (STROBE) statement: guidelines for reporting observational studies. PLoS Med 2007;4:e296. 


\section{Digital communication and sexual health}

23 Gilbert M, Bonnell A, Farrell J, et al. Click Yes to consent: acceptability of incorporating informed consent into an Internet-based testing program for sexually transmitted and blood-borne infections. Int J Med Inform 2017;105:38-48.

24 Salway T, Thomson K, Taylor D, et al. Post-test comparison of HIV test knowledge and changes in sexual risk behaviour between clients accessing HIV testing online versus in-clinic. Sex Transm Infect 2019;95:102-7.

25 Wilson E, Free C, Morris TP, et al. Can Internet-based sexual health services increase diagnoses of sexually transmitted infections (STI)? protocol for a randomized evaluation of an Internet-based STI testing and results service. JMIR Res Protoc 2016;5:e9.

26 Smith KS, Hocking JS, Chen MY, et al. Dual intervention to increase chlamydia retesting: a randomized controlled trial in three populations. Am J Prev Med 2015:49:1-11.
27 Kersaudy-Rahib D, Lydié N, Leroy C, et al. Chlamyweb Study II: a randomised controlled trial (RCT) of an online offer of home-based Chlamydia trachomatis sampling in France. Sex Transm Infect 2017;93:188-95.

28 Knight RE, Chabot C, Carson A, et al. Qualitative analysis of the experiences of gay, bisexual and other men who have sex with men who use GetCheckedOnline.com : a comprehensive internet-based diagnostic service for HIV and other STIs. Sex Transm Infect 2019;95:145-50.

29 Nyatsanza F, McSorley J, Murphy S, et al. 'It's all in the message': the utility of personalised short message service (SMS) texts to remind patients at higher risk of STIs and HIV to reattend for testing-a repeat before and after study. Sex Transm Infect 2016:92:393-5.

30 Bourne C, Knight V, Guy R, et al. Short message service reminder intervention doubles sexually transmitted infection/HIV re-testing rates among men who have sex with men. Sex Transm Infect 2011:87:229-31. 